\title{
IVD.IO - Usefulness of automated assays for detecting hepatitis B and C markers in dried blood spot samples
}

Livia Melo Villar ${ }^{1 *}$; Helena Medina Cruz ${ }^{1}$; Raissa Martins Deodato ${ }^{1}$; Juliana Custódio Miguel ${ }^{1}$; Geane Lopes Flores ${ }^{1}$; Lia Laura Lewis-Ximenez ${ }^{1}$; Elisabeth Lampe ${ }^{1}$.

1Fiocruz/IOC.

Introduction: Hepatitis $\mathrm{B}$ and $\mathrm{C}$ diagnosis are made by detecting antigens, antibodies or viral genome from serum samples obtained through venous blood collection. However, trained personnel and infrastructure to collect blood are required. Furthermore, low temperature storage conditions and transportation are necessary, which may be difficult to attain in limited-resource settings. Dried blood spots (DBSs) can be used to address these obstacles because they are easily collected and can be transported without refrigeration to reference laboratories for HCV diagnosis.

Objective: The present study was performed to evaluate the usefulness of electrochemiluminescence immunoassay "ECLIA" for anti-HCV, HBsAg and anti-HBc detection from DBS samples.

Methodology: Paired DBS and serum samples were obtained from individuals with or without hepatitis C or B that made up to Viral Hepatitis Laboratory repository. This study was approved by the Fiocruz Ethics Committee. DBS samples were prepared by spotting 3-5 drops (approximately $75 \mathrm{~mL}$ ) of whole blood from either a finger prick or a whole blood sample, collected by venipuncture without anticoagulant, onto Whatman filter paper 903 (GE Healthcare, USA), until 12-mm pre-printed circular paper disks were completely filled. To elute DBS samples, a 12-mm disc of filter paper was cut and transferred to a microtube containing $500 \mathrm{~mL}$ of PBS/BSA $0.5 \%$ at 18 to $24 \mathrm{hr}$. After incubation, eluate was directly submitted to the following ECLIA: Elecsys anti-HCV II, Elecsys HBsAg II and Elecsys anti-HBc II (Roche Diagnostics) following manufacturer's instructions. In the Anti-HCV and HBsAg assay, samples with sample/cutoff (S/CO) values $<1.0$ are considered non-reactive while for anti-HBc assay, non-reactive samples should present $\mathrm{S} / \mathrm{CO}>1.0$.

Results: Anti-HCV was detected in 103 DBS samples from 108 paired positive serum specimens and not detected in 364 DBS samples from 366 matched negative sera giving sensitivity of $95.4 \%$ and specificity of $99.4 \%$. HBsAg was reactive in 67 DBS out of 71 positive paired serum samples and was not detected among 295 DBS samples from 298 paired negative serum samples showing sensitivity and specificity of $94.4 \%$ and $99 \%$, respectively. Anti-HBc was detected in 160 DBS samples from 185 paired positive serum specimens and not detected in 349 DBS samples from 357 matched negative sera giving sensitivity of $86.5 \%$ and specificity of 97.8\%. Overall, the Kappa index indicated a high agreement between results obtained for the serum and DBS samples (k: 0.95, 0.93 and 0.86 for anti-HCV, HBsAg, anti-HBc, respectively).

Conclusion: In conclusion, ECLIA tested could be used for detecting hepatitis B and C markers in DBS. DBS specimens could be a reliable alternative testing specimen, which may increase hepatitis $\mathrm{B}$ and $\mathrm{C}$ diagnosing opportunities for rural, remote and hard to reach regions. DBS could be easily collected and transported to reference laboratories for testing using automated assays.

Keywords: hepatitis B; hepatitis C ; dried blood spot 